\title{
Impact of hydrogel and hyaluronic acid rectal spacer on rectal dosimetry and toxicity in low-dose-rate prostate brachytherapy: a multi-institutional analysis of patients' outcomes
}

\author{
Yuan-Hong Lin, BBiomedSc, MD!, Wee Loon, OngBMedSci, MBBS, MPhil (Epi)', Mark Tacey, MBiostat, BSc',2, \\ Damien Bolton, MD, MBBS, BA, FRACS, FRCS 3 , Alwin Tan, MBBS, FRACS ${ }^{4}$, Yee Chan, MBBS, FRACS ${ }^{3}$, Chee Wee Cham, \\ MBBS, FRCS, MD, FRACS ${ }^{4}$, Huong Ho, MSc, ApplSc $c^{5}$, Mario Guerrieri, MBBS, FRANZCR 5 , Farshad Foroudi, MBBS, MPA, \\ DMedSc, FRANZCR!' Daryl Lim Joon, MBBS, FRANZCR', Kevin McMillan, MBBS, BSc, FRACS ${ }^{6}$, George Koufogiannis, \\ MBBS, FRACS 6 , Paul Manohar, MBBS, FRACS 6 , Madalena Liu, MBBS, MD, FRACS , Trung Pham, MBBS, FRACS6, \\ Prof. Michael Chao, MBBS, FRANZCR, DMedS l,.5 \\ 'Department of Radiation Oncology, Austin Health, Heidelberg, Australia, ${ }^{2}$ Melbourne School of Population and Global Health, University \\ of Melbourne, Melbourne, Victoria, Australia, ${ }^{3}$ Department of Urology, Austin Health, Heidelberg, Australia, ${ }^{4}$ The Bays Hospital, Mornington, \\ Australia, ${ }^{5}$ Genesis Cancer Care Victoria, Ringwood East, Australia, ${ }^{6}$ Ringwood Private Hospital, Ringwood East, Australia
}

\begin{abstract}
Purpose: To report on rectal dosimetry and toxicity outcomes in men with prostate cancer (PCa) treated with iodine-125 low-dose-rate brachytherapy (LDR-BT) with or without polyethylene glycol hydrogel (HS) or hyaluronic acid (HA) rectal spacer (RS) insertion.

Material and methods: Seventy consecutive men treated with LDR-BT between December 2017 and July 2019 were included in this study, including twenty-eight (40\%) men who had RS insertion according to the preference of referring urologist, compared to a group of forty-two men (60\%) without RS. Descriptive statistics were used to compare RS safety, dosimetric effects on organs at risk (rectum and urethra), and gastrointestinal (GI) and genitourinary toxicities (GU) (assessed using the CTCAE v.4) between the two groups of patients.

Results: The median prostate-rectal separation with RS at mid prostate was $10 \mathrm{~mm}$ (IQR, 8-11.5 mm). The median follow-up was 23.5 months. There were no post-operative complications for RS insertion. There was significantly reduced rectal dosimetry in RS-group vs. non-RS group; the median $\mathrm{RV}_{100}$ was $0.0 \mathrm{cc}(\mathrm{IQR}, 0.0-0.0 \mathrm{cc}) \mathrm{vs} .0 .4 \mathrm{cc}$ (IQR, $0.1-1.1 \mathrm{cc})(p<0.001)$, respectively. The mean rectal $\mathrm{D}_{1 \mathrm{cc}}$ and $\mathrm{D}_{2 \mathrm{cc}}$ were $52.4 \%$ vs. $84.2 \%(p<0.001)$ and $45.7 \%$ vs. $70.0 \%$ $(p<0.001)$ for RS and non-RS group, respectively. There were no statistically significant differences in the mean urethral $\mathrm{D}_{20}, \mathrm{D}_{5}$, and $\mathrm{D}_{1}$. There were significantly less grade 1 acute and late GI toxicities in RS-group when compared to non-RS group ( $0 \%$ vs. $24 \%, p=0.004$ for acute GI toxicity; $4 \%$ vs. $33 \%, p=0.003$ for late GI toxicity). There were no reported acute or late grade 2 or above GI toxicities.

Conclusions: Insertion of RS in men treated with LDR-BT is safe and resulted in a significant reduction in rectal dosimetry. The reduction in rectal dosimetry with RS insertion translates into significantly reduced acute and late GI toxicities.

Key words: low-dose-rate brachytherapy, prostate cancer, rectal spacer, rectal toxicity, rectal dosimetry.

\section{Purpose}

Low-dose-rate brachytherapy (LDR-BT) is an effective curative treatment for low- and intermediate-risk localized prostate cancer (PCa) [1], and as salvage radiotherapy with curative intent in localized recurrence [2]. How- ever, the risk of rectal toxicities poses a major challenge to prostate irradiation. This was demonstrated in a large retrospective study, which reported an incidence of $20.57 \%$ grade 1 proctitis and $6.4 \%$ grade 2 proctitis, following individual or combination BT [3]. Significantly, von Gellekom et al. demonstrated a correlation between increasing
Address for correspondence: Prof. Michael Chao, Ringwood Private Hospital, 36 Mount Dandenong Road, Ringwood VIC 3135, phone: +61 3 88703300, fax: +61 388703388 ,

e-mail: Michael.Chao@genesiscare.com
Received: 23.04 .2021

Accepted: 19.09 .2021

Published: 30.12.2021 
rectal $\mathrm{D}_{2 \mathrm{cc}}$ with an increasing incidence of rectal toxicity [4]. Snyder et al. also reported a reduced rate of $\geq$ grade 2 rectal toxicity, if $\mathrm{V}_{100}$ to the rectum was minimized to $<1.3$ cc [5]. Peters et al. further recommended minimizing $\mathrm{D}_{2 \mathrm{cc}}$ to less than $100 \mathrm{~Gy}$ in whole gland salvage iodine- 125 $\left({ }^{125} \mathrm{I}\right) \mathrm{BT}$ prostate permanent implant to minimize severe gastrointestinal (GI) toxicities [6]. Furthermore, Veccia et al. demonstrated that reducing post-LDR-BT implant rectal $\mathrm{V}_{100}\left(\mathrm{RV}_{100}\right)$ to $\leq 0.5 \mathrm{cc}$, yielded improved acute and chronic rectal function and patient's quality of life [7]. All current literature demonstrated a need to minimize both rectal dose and rectal volume receiving maximum irradiation in order to avoid severe GI complications.

The use of a bio-degradable recto-prostatic spacer has been widely studied over the past decade and examining its' utility to increase the distance between prostate and rectum and thus, minimizing radiation dose received by the rectum. Currently available options for recto-prostatic spacer include hyaluronic acid (HA), implanted balloons [8], collagen implants [9], and polyethylene glycol hydrogel (HS). Hyaluronic acid, HS, and bio-degradable implanted balloons all demonstrated promising preliminary results in reducing rectal dosimetry [10-13] and decreasing rates of acute and chronic rectal toxicities $[14,15]$.

Both HS and HA rectal spacers present as cost-effective [16], safe, and effective recto-prostatic spacers. Current evidence suggests a significant reduction in radiation dose to the rectum in HS patients undergoing LDR-BT with or without external beam radiotherapy (EBRT), when compared to patients without hydrogel injection [17-20]. Furthermore, HS is associated with minimal post-operative complications and low rates of grade $\geq 3$ acute or late rectal toxicities [13, 21-25]. Despite the promising findings from the literature, there have been a lack of large scale prospective clinical trials conducted for HS or HA rectal spacers in LDR-BT. As a result, this retrospective multi-institutional cohort study aims to report the rectal dosimetry and toxicity outcomes in men with PCa treated with LDR-BT with or without HS or HA rectal spacer (RS) insertion.

\section{Material and methods}

\section{Study design, setting, and ethics}

This retrospective case-control cohort study, assessing a prospective database, was performed to evaluate the efficacy and safety of HS or HA RS in LDR-BT for patients with clinically localized low-risk and intermediate-risk PCa at a private radiation oncology center (Genesis Care) in Melbourne, Australia. Ethics approval was sourced from the above institution on March 5, 2018. Between October 2017 and July 2019, 70 consecutive patients were enrolled into this study. LDR-BT prescription was 145 Gy modified peripherally loading (MPD) using ${ }^{125} \mathrm{I}$ as monotherapy or with downsizing androgen deprivation therapy (ADT). The use of HS or HA RS was based on the preference of treating physician (i.e., urologist) only. HS RS (SpaceOAR ${ }^{\circledR}$, Boston Scientific, MA, USA) was exclusively used for insertion prior to 2020, whilst HA RS (Barrigel ${ }^{\circledR}$, Palette Life Sciences, Stockholm Swe- den) was preferred following 2020, as it became commercially available in Australia.

All patients were initially assessed with a thorough medical history, examination, and serum prostate specific antigen (PSA) level. A digital rectal examination (DRE) was performed to evaluate patient's clinical T-stage (cT). Transperineal ultrasound-guided biopsy (TPUS) was performed and assessed by an accredited general pathologist to produce pathological staging with either Gleason score or International Society of Urological Pathology (ISUP) score. All patients with Gleason score $>7$ was staged with computerized tomography (CT) scan of abdomen and pelvis, magnetic resonance imaging (MRI) prostate, and/or whole-body bone scan (WBBS).

Prostate cancer stratification was calculated based on D'Amico classification [26]. Low-risk PCa was defined as Gleason score $\leq 6$, PSA $\leq 10 \mathrm{ng} / \mathrm{ml}$, and stage cT1-cT2a; favorable and unfavorable intermediate-risk PCa were defined as Gleason score of 7 (favorable intermediate-risk group was defined as Gleason $3+4$ grade group 2, whilst unfavorable risk group was defined as Gleason $4+3$ grade group 3), and/or stage cT2b PCa. Patients without a specified cT2 sub-stage but with Gleason score $\leq 6$ and PSA $<10$, were reclassified as low-risk rather than left unclassified.

Gastrointestinal and genitourinary (GU) toxicities were assessed using common terminology criteria for adverse events (CTCAE) version 4.0. Acute GI and GU toxicities were defined as symptom occurrence within 3 months post-treatment, whilst late toxicities were described as symptom occurrence after 3 months post-treatment. Immediate or delayed post-operative complications following RS insertion, including, but not limited to, rectal tear, rectal perforation, rectal ulceration, rectal bleeding, infection, allergic reactions, and urinary retention were recorded in electronic medical records and/or on patient's progress note at their first post-treatment follow-up with radiation oncologist. Assessing physicians were not blinded to the use of RS.

\section{Planning and treatment}

This study implemented the same volume study, treatment planning, and treatment techniques as previously reported by Chao et al. [27].

Any patient who underwent a pre-implant TURP had limited resection with only 3-5 grams of tissue removed. If an intravesical median lobe was present, the lobe was resected down to prostatic capsule. In some cases, patients also had a transurethral incision of the prostate (TUIP). Patients did not proceed to their implant until at least 3 months after their TURP, with urodynamic tests and cystoscopy performed to confirm resolution of outlet obstruction and urethral healing.

Rectal spacer insertion with either HS (SpaceOAR ${ }^{\circledR}$, Boston Scientific, MA, USA) or HA (Barrigel ${ }^{\circledR}$, Palette Life Sciences, Stockholm, Sweden) was performed at the end of an implant via transrectal ultrasound guidance by the treating radiation oncologist or urologist. 10 cc of Space$\mathrm{OAR}^{\odot}$ hydrogel spacer, or $9 \mathrm{cc}$ of Barrige ${ }^{\odot}$ hyaluronic $^{\circ}$ acid gel was introduced into the recto-prostatic space. RS insertion was performed at the end of the implant, as the 
use of RS could degrade the images of the prostate, therefore interfere with the implant of brachytherapy rods. It could also potentially elevate the prostate anteriorly and cause pubic arch obstruction.

Patients were admitted overnight with an indwelling catheter (IDC) and discharged after a successful trial of void in the following morning. All patients attended a follow-up with the urologist at 2 weeks post-implantation as well as a follow-up with the radiation oncologist at 4 weeks post-implantation for post-implant dosimetry using CT/MRI to verify for post-procedural complications. Placement of RS and the resultant prostate-rectum separation was characterized using MRI.

The patients were then followed-up every 3 to 4 months for the first year by the treating radiation oncologist, and every 6 months for the next 5 years. PSA testing was performed for at least first 4 years of follow-up, with biochemical failure defined as PSA nadir $+2 \mathrm{ng} / \mathrm{l}$, following implant and exclusion of PSA bounce according to Phoenix definition [28].

\section{Statistical analyses}

Descriptive statistics were performed to evaluate characteristics of the patients demographics, disease, treatment features, and post-treatment toxicities, and test for differences between groups. Continuous variables were presented as mean \pm standard deviation or median (interquartile range) for normal and non-normal variables, respectively, using Student's $t$-test and Mann-Whitney test for differences between the two groups. Categorical variables were presented as counts and percentage frequencies, with chi-square or Fisher's exact test applied to evaluate differences in distribution between the groups. Analysis of the association between $\mathrm{RV}_{100}$ with acute and late GI toxicities included categorizing $\mathrm{RV}_{100}$ based on thresholds of $\leq 0.5,>0.5, \leq 1.3$, and $>1.3$ as per previously published dose constraints $[5,7$, $29,30]$. Evaluations of association between pre- and post-implant rectal $\mathrm{D}_{1 \mathrm{cc}}$ and $\mathrm{D}_{2 \mathrm{cc}}$ relations with GI toxicities included consideration of medians and/or thresholds, as determined by utilization of receiver operating curve (ROC) analysis. Potential confounding effects of $R V_{100}, D_{1 c c}$ and $D_{2 c c}$ values on the association between RS and late GI toxicity was explored using multi-variable logistic regression analysis. Multi-variable analysis could not be explored for acute GI toxicity due to no acute GI toxicity events in the RS cohort. Statistical analysis was conducted using Stata version 15.1 (StataCorp, College Station, Texas, USA), with statistical significance defined as $p$-value $<0.05$.

\section{Results}

\section{Patients' characteristics}

The mean age of the study population was 66.1 years $(\mathrm{SD}=6.8)$ (Table 1). Of the 70 men enrolled in the study, 28 patients $(40 \%)$ received RS insertion and 42 men $(60 \%)$ did not receive RS. The median follow-up was 23.5 months (IQR = 20.75 months). It was 26.5 months $(\mathrm{IQR}=17.5$ months) for the patients who did not receive
RS, and 13 months (IQR = 20.5 months) for the patients who received RS. There were 19 men $(27 \%)$ with lowrisk $\mathrm{PCa}, 49$ (70\%) with favorable intermediate-risk PCa, and 3 cases $(4 \%)$ with unfavorable intermediate-risk $\mathrm{PCa}$, with a statistically significant difference in cT-stages in patients with RS vs. patients without RS insertion $(p=0.025)$. Overall, 43 men $(61 \%)$ underwent TURP prior to LDR-BT, whilst 9 men (13\%) received downsizing ADT, with no statistically significant differences between the cohorts. The high volume of TURP was attributed to poor urinary flow and was performed at the discretion of the treating urologist with small volume resection (3-5 grams of tissues removed) for patients with poor flow.

There was no statistically significant difference in the mean pre-radiotherapy (RT) PSA in patients treated with RS vs. non-RS group $(5.0, \mathrm{SD}=2.6 ; 4.9, \mathrm{SD}=2.4 ; p=0.91)$, or distribution in Gleason score $(p=0.53)$ or ISUP grading $(p=0.53)$.

There was no statistically significant difference in the number of patients treated with RS vs. patients not treated with RS (staging scan performed for almost all patients), who underwent MRI imaging ( $p=0.64)$, CT staging $(p=1.00)$, WBBS $(p=1.00)$, or downsizing androgen deprivation therapy (ADT) $(p=1.00)$.

No PSA relapse was recorded in any men. The median follow-up PSA was 0.3 (IQR, 0.1-0.6) for men treated without RS, and 0.6 (IQR, 0.3-1.0) for men treated with RS $(p=0.019)$.

\section{Dosimetry results}

The mean prostate volume was $37.7 \mathrm{cc}(\mathrm{SD}=9.1)(\mathrm{Ta}-$ ble 2), with no statistically significant difference in the mean prostate volume recorded between men with RS vs. men without RS ( $p=0.15$; Table 2$)$. The mean number of seeds inserted was $70.7(\mathrm{SD}=7.9)$, with no differences between men with RS vs. men without RS $(p=0.42)$. No post-procedural insertion complications were recorded.

No statistically significant difference was recorded for the mean urethral $D_{20}, D_{5}$, and $D_{1}$ for men without RS insertion vs. men with RS insertion: $130.8 \%$ vs. $129.4 \%$ $(p=0.20), 137.1 \%$ vs. $135.5 \%(p=0.22)$, and $145.0 \%$ vs. $141.5 \%(p=0.061)$, respectively. Similarly, no statistically significant difference was recorded amongst the two groups for pre-RS insertion prostate $\mathrm{D}_{90}, \mathrm{~V}_{100}, \mathrm{~V}_{150}$, and $\mathrm{V}_{200}$, with $119.6 \%$ vs. $119.4 \%$ ( $\left.p=0.62\right), 98.6 \%$ vs. $98.6 \%$ $(p=0.42), 55.5 \%$ vs. $55.4 \%(p=0.90)$, and $23.1 \%$ vs. $22.7 \%$ $(p=0.48)$, respectively, as well as for post-RS insertion prostate $\mathrm{D}_{90}, \mathrm{~V}_{100}, \mathrm{~V}_{150}$, and $\mathrm{V}_{200}$, with $98.5 \%$ vs. $99.6 \%$ $(p=0.66), 88.6 \%$ vs. $88.7 \%(p=0.93), 50.3 \%$ vs. $48.9 \%$ $(p=0.58)$, and $23.6 \%$ vs. $22.0 \%(p=0.61)$, respectively.

The median recto-prostatic separation, as measured from midline of the prostate, was $10 \mathrm{~mm}(\mathrm{IQR}=3.25 \mathrm{~mm})$ in the patients who underwent RS. There was significantly reduced rectal dosimetry in men with RS vs. men without RS, i.e., $\mathrm{RV}_{100}=0 \mathrm{cc}(\mathrm{IQR}=0-0.0 \mathrm{cc})$ vs. $\mathrm{RV}_{100}=0.4 \mathrm{cc}$ $(\mathrm{IQR}=0.1-1.1 \mathrm{cc}), p<0.001$. Also, there was significantly reduced $D_{1 c c}$ and $D_{2 c c}$ in men with RS vs. men without RS. $\mathrm{D}_{1 \mathrm{cc}}$ in men with $\mathrm{RS}$ was $52.4 \%$ vs. $84.2 \%$ in men without RS $(p<0.001)$. $D_{2 c c}$ was $45.7 \%$ in men with RS vs. $70.0 \%$ in men without RS $(p<0.001)$. 
Table 1. Patients' characteristics (overall, RS vs. non-RS group)

\begin{tabular}{|c|c|c|c|c|}
\hline Factor & Overall & Patients without RS & Patients with RS & $p$-value \\
\hline$n$ & 70 & 42 & 28 & \\
\hline Age, mean (SD) & $66.1(6.8)(n=69)$ & $66.9(7.0)$ & $64.9(6.3)(n=27)$ & 0.25 \\
\hline Follow-up (months), median (IQR) & $23.5(20.75)$ & $26.5(17.5)$ & $13(20.5)$ & \\
\hline Pre-RT PSA, mean (SD) & $5.0(2.5)$ & $5.0(2.6)$ & $4.9(2.4)$ & 0.91 \\
\hline cT stage, $n(\%)$ & & & & 0.025 \\
\hline T1c & $19(27)$ & $7(17)$ & $12(43)$ & \\
\hline $\mathrm{T} 2 \mathrm{a}$ & $49(70)$ & $34(81)$ & $15(54)$ & \\
\hline $\mathrm{T} 2 \mathrm{~b}$ & $2(3)$ & $1(2)$ & $1(4)$ & \\
\hline Gleason score, $n$ (\%) & & & & 0.53 \\
\hline $3+3$ & $25(36)$ & $14(33)$ & $11(39)$ & \\
\hline $3+4$ & $42(60)$ & $27(64)$ & $15(54)$ & \\
\hline $4+3$ & $3(4)$ & $1(2)$ & $2(7)$ & \\
\hline ISUP, $n(\%)$ & & & & 0.53 \\
\hline 1 & $25(36)$ & $14(33)$ & $11(39)$ & \\
\hline 2 & $42(60)$ & $27(64)$ & $15(54)$ & \\
\hline 3 & $3(4)$ & $1(2)$ & $2(7)$ & \\
\hline Cores positive, mean (SD) & $0.3(0.2)$ & $0.2(0.2)$ & $0.3(0.2)$ & 0.030 \\
\hline Risk classification, $n$ (\%) & & & & 0.44 \\
\hline Low & $24(34)$ & $13(31)$ & $11(39)$ & \\
\hline Favorable IR & $43(61)$ & $28(67)$ & $15(54)$ & \\
\hline Unfavorable IR & $3(4)$ & $1(2)$ & $2(7)$ & \\
\hline TURP, $n(\%)$ & $43(61)$ & $29(69)$ & $14(50)$ & 0.14 \\
\hline MRI, $n(\%)$ & $65(93)$ & $38(90)$ & $27(96)$ & 0.64 \\
\hline Downsizing ADT, $n(\%)$ & $9(13)$ & $6(14)$ & $3(11)$ & 1.00 \\
\hline \multicolumn{5}{|l|}{ PSA relapse } \\
\hline No & $42(100 \%)$ & $28(100 \%)$ & & \\
\hline PSA, median (IQR) & $0.3(0.1,0.6)(n=42)$ & $0.6(0.3,1.0)(n=28)$ & 0.019 & \\
\hline
\end{tabular}

\section{Immediate and late GI and GU toxicities}

There was a statistically significant difference in acute GI toxicity of $24 \%$ to $0 \%(p=0.004)$ and in late GI toxicity of $33 \%$ to $4 \%(p=0.003)$ in the non-RS vs. RS cohorts (Table 3). The only late GI toxicity in the RS cohort was observed in one patient with rectal bleeding. As shown in Table 3, the most common late GI toxicities observed in the non-RS cohort were grade 1 diarrhea, proctitis, and rectal bleeding. No acute or late grade 2 or higher GI toxicities were observed in either group.

Table 4 demonstrates further analyses of the association between $\mathrm{RV}_{100}, \mathrm{D}_{1 \mathrm{cc}}$ and $\mathrm{D}_{2 \mathrm{cc}}$ with acute and late GI toxicities. There was a statistically significant difference observed between patients with post-implant $\mathrm{RV}_{100} \leq 0.5$ and $>0.5$ and late GI toxicity ( $15 \%$ vs. $39 \%, p=0.049$ ). As there were no pre-implant $\mathrm{RV}_{100}$ values beyond 1.3, no assessment of association with acute GI toxicity could be performed. Rates of acute GI toxicity were higher in post-implant $\mathrm{RV}_{100}$ values $>1.3$ compared to $\leq 1.3(29 \%$ vs. $13 \%)$ and for late GI toxicity ( $29 \%$ vs. $21 \%)$, but neither comparison was statistically significant $(p=0.26$ and 0.64 , respectively).
Based on ROC analysis, acute GI toxicity was higher for patients with post-implant rectal $D_{1 \mathrm{cc}}$ values $>67.45$ $(25 \%$ vs. $3 \%, p=0.014)$, with late GI toxicity also higher $(36 \%$ vs. $6 \%, p=0.003)$. Similarly, acute GI toxicity rates were higher for post-implant rectal $\mathrm{D}_{2 \mathrm{cc}}$ values $>53.89$ $(23 \%$ vs. $3 \%, p=0.035)$, with a cut-point $>54.81$ maximizing the differential in late GI toxicity (35\% vs. $6 \%$, $p=0.003$ ).

Univariate logistic regression analysis provided odds ratio of 0.07 ( $95 \%$ CI: $0.01-0.60 \%, p=0.15$ ) for $\log$ odds of late GI toxicity in the RS cohort, when compared to the non-RS cohort. After adjusting for post-implant $\mathrm{RV}_{100}$ $>0.5$, statistical significance was maintained with a slightly higher odds ratio $(\mathrm{OR}=0.09 ; 95 \% \mathrm{CI}$ : $0.01-0.80 \%$; $p=0.030)$. When adjusting for post-rectal $\mathrm{D}_{1 \mathrm{cc}}$ and $\mathrm{D}_{2 \mathrm{cc}}$ in separate models, statistical significance was not retained. Although, ORs were all in the order of $<0.2\left(\mathrm{D}_{1 \mathrm{cc}}\right.$ : OR $=$ $0.19,95 \%$ CI: $0.01-2.70 \%, p=0.22 ; \mathrm{D}_{2 \mathrm{cc}}$ : $\mathrm{OR}=0.16,95 \%$ CI: $0.01-1.80 \%, p=0.14$ ), indicating that the association between RS and late GI toxicity was only marginally affected by confounding, and a strong association between RS and late GI toxicity remained. 


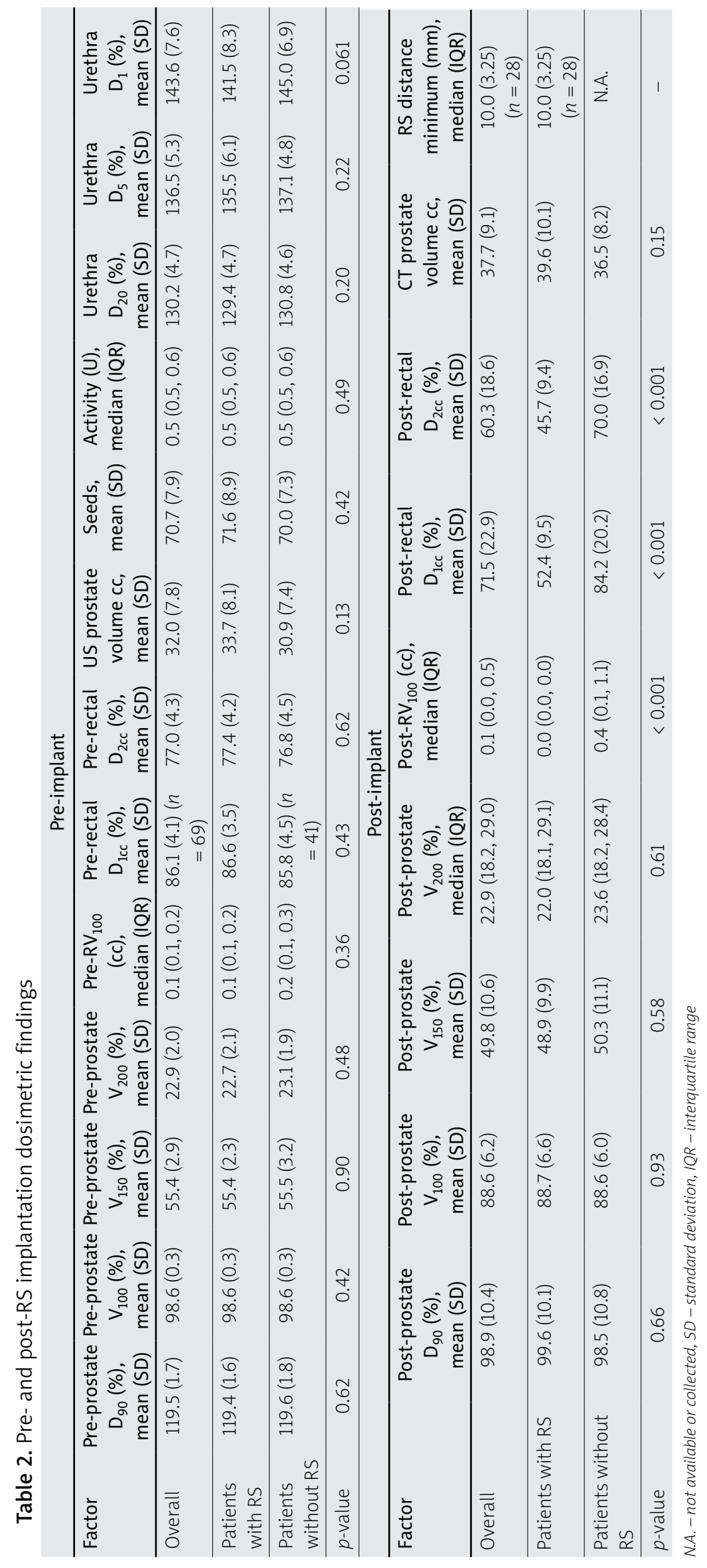


Table 3. Acute and late GU and GI toxicities in patients treated with RS vs. no RS

\begin{tabular}{lccc}
\multicolumn{1}{l}{ Toxicity type and grade } & Patients without RS $(n=42)$ & Patients with RS $(n=28)$ & $p$-value \\
\hline \begin{tabular}{l} 
Acute toxicities, $n(\%)$ \\
\hline Any GU toxicity
\end{tabular} & $37(88)$ & $24(86)$ & 1.00 \\
\hline Any grade 2+ GU toxicity & $2(5)$ & $1(4)$ & 0.81 \\
\hline Any GI toxicity & $10(24)$ & $0(0)$ & 0.004 \\
\hline Any grade 2+ Gl toxicity & $0(0)$ & $0(0)$ & 1.00 \\
\hline Late toxicities, $n(\%)$ & $27(64)$ & $18(64)$ & 1.00 \\
\hline Any late GU toxicity & $4(10)$ & $1(4)$ & 0.64 \\
\hline Any grade 2+ GU toxicity & $14(33)$ & $1(4)$ & 0.003 \\
\hline Any late Gl toxicity & $0(0)$ & $0(0)$ & 1.00
\end{tabular}

Table 4. Dosimetric association with acute and late toxicities

\begin{tabular}{|c|c|c|c|c|c|c|}
\hline \multirow[t]{2}{*}{ Variable } & \multicolumn{3}{|c|}{ Acute GI toxicity } & \multicolumn{3}{|c|}{ Late GI toxicity } \\
\hline & No & Yes & $p$-value & No & Yes & $p$-value \\
\hline Group & & & 0.004 & & & 0.003 \\
\hline Non-RS cohort & $32(76.2)$ & $10(23.8)$ & & $28(66.7)$ & $14(33.3)$ & \\
\hline RS cohort & $28(100.0)$ & $0(0)$ & & $27(96.4)$ & $1(3.6)$ & \\
\hline Pre- $\mathrm{RV}_{100}(\mathrm{cc})$ & & & 0.146 & & & 0.58 \\
\hline$\leq 0.5$ & $57(87.7)$ & $8(12.3)$ & & $50(76.9)$ & $15(23.1)$ & \\
\hline$>0.5$ & $3(60.0)$ & $2(40.0)$ & & $5(100.0)$ & 0 & \\
\hline \multicolumn{7}{|l|}{ Pre-RV ${ }_{100}(\mathrm{cc})$} \\
\hline$\leq 1.3$ & $60(85.7)$ & $10(14.3)$ & & $55(78.6)$ & $15(21.4)$ & \\
\hline$>1.3$ & $0(0)$ & $0(0)$ & & $0(0)$ & $0(0)$ & \\
\hline Pre-rectal $\mathrm{D}_{1 \mathrm{cc}}(\%)$ & & & 0.74 & & & 0.77 \\
\hline$\leq 86.60$ (median and ROC) & $31(83.8)$ & $6(16.2)$ & & $30(81.1)$ & 7 (18.9) & \\
\hline$>86.60$ (median and ROC) & $29(87.9)$ & 4 (12.1) & & $25(75.8)$ & $8(24.2)$ & \\
\hline Pre-rectal $D_{2 c c}(\%)$ & & & 0.18 & & & 0.39 \\
\hline$\leq 77.08$ (median and ROC) & $33(91.7)$ & $3(8.3)$ & & $30(83.3)$ & $6(16.7)$ & \\
\hline$>77.08$ (median and ROC) & $27(79.4)$ & $7(20.6)$ & & $25(73.5)$ & $9(26.5)$ & \\
\hline Post-RV 100 (cc) & & & 0.71 & & & 0.049 \\
\hline$\leq 0.5$ & $45(86.5)$ & $7(13.5)$ & & $44(84.6)$ & $8(15.4)$ & \\
\hline$>0.5$ & $15(83.3)$ & $3(16.7)$ & & $11(61.1)$ & $7(38.9)$ & \\
\hline Post-RV 100 (cc) & & & 0.26 & & & 0.64 \\
\hline$\leq 1.3$ & $55(87.3)$ & $8(12.7)$ & & $50(79.4)$ & $13(20.6)$ & \\
\hline$>1.3$ & $5(71.4)$ & $2(28.6)$ & & $5(71.4)$ & $2(28.6)$ & \\
\hline Post-rectal $\mathrm{D}_{1 \mathrm{cc}}(\%)$ & & & 0.014 & & & 0.003 \\
\hline$\leq 67.45(\mathrm{ROC})$ & $33(97.1)$ & $1(2.9)$ & & $32(94.1)$ & $2(5.9)$ & \\
\hline$>67.45$ (ROC) & $27(75.0)$ & $9(25.0)$ & & $23(63.9)$ & $13(36.1)$ & \\
\hline Post-rectal D 2 cc $(\%)$ & & & 0.035 & & & \\
\hline$\leq 53.89(\mathrm{ROC})$ & $30(96.8)$ & $1(3.2)$ & & & & \\
\hline$>53.89$ (ROC) & $30(76.9)$ & $9(23.1)$ & & & & \\
\hline Post-rectal $D_{2 c c}(\%)$ & & & & & & 0.003 \\
\hline$\leq 54.81(\mathrm{ROC})$ & & & & 31 (93.9) & $2(6.1)$ & \\
\hline$>54.81(\mathrm{ROC})$ & & & & $24(64.9)$ & $13(35.1)$ & \\
\hline
\end{tabular}

$R O C$ - cut-point as defined by receiver operating curve $(R O C)$ analysis 


\section{Discussion}

\section{Safety analyses}

This retrospective multi-institutional study demonstrated that RS insertion with HA or HS spacers were safe, with no post-operative complications identified. This remained consistent with safety analysis performed by Heikkilä et al. and Prada et al. for HS and HA spacer injections, respectively, with both studies showing no post-operative complications $[17,31]$. Furthermore, a systematic review performed by Miller et al. showed a $97 \%$ success rate with HS insertion and a low rate of procedural complication (0-10\% of patients), which were mild and transient in nature [32].

\section{RS distance}

The median distance of separation achieved in this cohort remained consistent to that reported in the literature. Similar recto-prostatic separations were described in intensity-modulated radiation therapy (IMRT) [19], LDR-BT, and HDR-BT using either HS or HA insertions $[14,21,22,33]$. Significantly two systematic reviews have established similar prostate-rectum separation with HS insertion in EBRT, BT, and combination RT (11.2 $\mathrm{mm})$ [32] as well as in LDR-BT and HDR-BT $(10 \mathrm{~mm})$ with that of the current study [25].

\section{Dosimetry outcomes}

The present study showed comparable prostate and urethral dosimetry measures for the cohort treated with RS and that not treated with RS. The post-implant constraints and dose goals were consistent with guidelines laid out by the American Association of Physicists in Medicine (AAPM) and the American Brachytherapy Society $[29,34]$. Most significantly, rectal dosimetry measures were significantly reduced in the RS cohort when compared to the non-RS cohort. As outlined above, there was a significant reduction in $\mathrm{RV}_{100}$ post-RS insertion $(0.4 \mathrm{cc}$ in the non-RS cohort vs. 0.0 cc in the RS cohort; $p<0.001$ ). The mean rectal $D_{1 c c}$ and $D_{2 c c}$ were both significantly reduced in patients with RS compared to the non-RS cohort (52.4\% vs. $84.2 \%$ for $\mathrm{D}_{1 \mathrm{cc}}$ and $45.7 \%$ vs. $70.0 \%$ for $\mathrm{D}_{2 \mathrm{cc}}$; $p<0.001)$.

Reduced rectal dosimetry following RS insertion was widely reported in high-dose-rate brachytherapy (HDRBT), includingChaoetal.study, whodemonstrated reduced rectal dose volumes from rectal $V_{30}$ to rectal $V_{80}$ following HS insertion [33,35]. Strom et al. observed that HS insertion in patients treated with HDR-BT with or without IMRT, demonstrated significantly reduced mean rectal D2 ml (47 $\pm 9 \%$ ) when compared to those without HS insertion $(60 \pm 8 \%)$, with values similar to those reported in this study [36]. Significantly, Taggar et al. showed reduced $\mathrm{D}_{1 \mathrm{cc}}$ and $D_{2 c c}$ values for HS insertion in patients treated with LDR-BT with palladium-103 as monotherapy, with EBRT, or as salvage monotherapy, when compared to patients not treated with HS [13]. HA insertion was demonstrated to reduce rectal dosimetry in HDR-BT with EBRT and HDR-BT in gynecological cancers $[31,38]$. In the setting
Table 5. Multi-variable analyses

Multi-variable, adjusted for post- $\mathrm{RV}_{100}$ (cc) $>0.5$

\begin{tabular}{|c|c|c|c|}
\hline \multirow[t]{2}{*}{ Variable } & \multicolumn{3}{|c|}{ Late GI toxicity } \\
\hline & OR & $95 \% \mathrm{Cl}$ & $p$-value \\
\hline \multicolumn{4}{|l|}{ Group } \\
\hline Non-RS cohort & 1.00 & & \\
\hline RS cohort & 0.09 & $0.01 \%$ to $0.80 \%$ & 0.03 \\
\hline \multicolumn{4}{|l|}{ Post-RV $100(\mathrm{cc})$} \\
\hline$\leq 0.5$ & 1.00 & & \\
\hline$>0.5$ & 1.55 & $0.42 \%$ to $5.63 \%$ & 0.51 \\
\hline \multicolumn{4}{|c|}{ Multi-variable, adjusted for rectal $D_{1 c c}(\%)>67.45$} \\
\hline \multirow[t]{2}{*}{ Variable } & & Late GI toxicity & \\
\hline & OR & $95 \% \mathrm{Cl}$ & $p$-value \\
\hline \multicolumn{4}{|l|}{ Group } \\
\hline Non-RS cohort & 1.00 & & \\
\hline RS cohort & 0.19 & $0.01 \%$ to $2.70 \%$ & 0.22 \\
\hline \multicolumn{4}{|l|}{ Post-rectal $D_{1 c c}(\%)$} \\
\hline$\leq 67.45$ & 1.00 & & \\
\hline$>67.45$ & 3.14 & $0.40 \%$ to $24.45 \%$ & 0.28 \\
\hline \multicolumn{4}{|c|}{ Multi-variable, adjusted for rectal $D_{2 c c}(\%)>54.81$} \\
\hline \multirow[t]{2}{*}{ Variable } & & Late GI toxicity & \\
\hline & OR & $95 \% \mathrm{Cl}$ & $p$-value \\
\hline \multicolumn{4}{|l|}{ Group } \\
\hline Non-RS cohort & 1.00 & & \\
\hline RS cohort & 0.16 & $0.01 \%$ to $1.80 \%$ & 0.14 \\
\hline \multicolumn{4}{|l|}{ Post-rectal $D_{2 c c}(\%)$} \\
\hline$\leq 54.81$ & 1.00 & & \\
\hline$>54.81$ & 3.19 & $0.50 \%$ to $20.34 \%$ & 0.22 \\
\hline
\end{tabular}

$O R$ - odds ratio

of IMRT, a phase 3 multicenter randomized clinical trial has been performed with HS insertion, yielding a significant reduction in rectal $\mathrm{V}_{70}$ post-spacer insertion [39-41]. Another clinical trial by van Gysen et al. was demonstrated a significant reduction in rectal doses $\left(\mathrm{V}_{30}\right.$ to $\left.\mathrm{V}_{80}\right)$ post-HS insertion in 10 patients treated with volumetric modulated arc therapy (VMAT) [42]. These findings suggest that HS insertion produces a consistent reduction in rectal dosimetry across different radiotherapy settings.

Placement of HS in patients treated with ${ }^{125}$ I LDR-BT was demonstrated by Morita et al., showing reduced $\mathrm{RV}_{150}$ and $\mathrm{RV}_{100}$ compared with those not treated with HS [43]. Furthermore, a systematic review, which included 12 studies of HS insertion in the setting of LDR-BT and HDR-BT with or without EBRT, VMAT, or IMRT demonstrated that rectal doses were reduced across all these different settings [25]. Further studies by Kahn et al., Butler et al., Patel et al., Taggar et al., Zhang et al., and Liu et al. all showed improvements in rectal dosimetry post-HS insertion for patients treated with LDR-BT with or without EBRT [13, 44-49]. As such, the reduced rectal dosimetry post-RS insertion in the present study were comparable to, and reaffirming findings from, current literature on LDR-BT. 


\section{Toxicity outcomes}

There was no statistically significant difference in acute or late grade 1 to $3 \mathrm{GU}$ toxicities between the RS group and the non-RS group (Table 3). No acute or late grade 4 GU toxicities were recorded in this study. Similar incidence of GU toxicities were reported by Uhl et al., where HS cohort experienced $41.7 \%$ acute grade 1, 35.4\% grade 2 , and $2.1 \%$ grade 3 GU toxicities, and $17 \%$ late grade 1 and $2.1 \%$ late grade $2 \mathrm{GU}$ toxicities [50]. Chao et al. reported no statistically significant difference in acute or late GU toxicities between group treated with HS and group not treated with HS in HDR-BT with or without EBRT [33]. This study showed that RS insertion did not increase the incidence of acute or late $\geq$ grade 1 GU toxicities, with reported incidences comparable to the current literature.

The present study demonstrated a statistically significant reduction in acute and late GI toxicities post-RS insertion, which were supported by findings of the literature. Wilder et al. showed that HA insertion yielded an incidence of $0 \%$ acute grade 1 to 3 acute diarrhea in 10 treated patients following IMRT and HDR-BT, when compared to an incidence of $29.7 \%$ in patients who did not receive HA [51]. Taggar et al. reported an incidence of 9.5\% grade 1 rectal discomfort or rectal bleeding post-HS insertion for LDR-BT with or without EBRT [13]. A systematic review performed by Vaggers et al. on HS use in prostate brachytherapy described an acute grade 1 or 2 GI toxicity rate of $33.7 \%$ and a low-rate of grade 3 or 4 GI complications $(0.22 \%)$ [52]. In the Australian setting, Chao et al. reported a statistically significant reduction in acute $\geq$ grade 1 GI toxicity in HS patients treated with HDR-BT and EBRT compared to non-HS group [33]. Low rates of late grade 1 GI toxicity in both groups were reported ( $0 \%$ in HS group and $7.7 \%$ in non-HS group, $p=0.11$ ). A systematic review and meta-analysis by Miller et al., who included patients treated with BT, EBRT, or combination therapy, showed that reduced rectal dosimetry in HS-treated group translated to a significant reduction in late grade 2 or above GI toxicities as well as significant improvement in bowel-related quality of life at late follow-up [32]. Another systematic review by Ardekani et al. focused on patients treated with LDR-BT or HDR-BT also demonstrated a reduction in acute and late GI toxicities post-HS insertion [25]. Furthermore, several other research have also reported significant reduction in acute and late rectal toxicities post-RS insertion in LDR-BT as monotherapy, or as combination therapy [13, 45, 46, 53] in HDR-BT [25, 32, 35, 37, 54, 55], and particularly in the settings of IMRT in large-scale multi-institutional randomized clinical trial [39-41].

Statistical analyses presented in Tables 4 and 5 further support a strong correlation between the reduction of rectal dosimetry $\left(\mathrm{RV}_{100}\right.$, rectal $\mathrm{D}_{1 \mathrm{cc}}$ and $\left.\mathrm{D}_{2 \mathrm{cc}}\right)$ and the reduction in acute or late GI toxicities. Significantly, reducing $\mathrm{RV}_{100}$ to $\leq 0.5$ yielded a lowered rate of late GI toxicity, when compared to the cohort of patients who received $R_{100}>$ 0.5. This was consistent with findings from Veccia et al. who reported improvements in acute and late rectal functions when post-implant $\mathrm{RV}_{100}$ was restricted to $\leq 0.5$ [7].
Similarly, Shiraishi et al. also reported a reduction in rates of grade 2 rectal bleeding in patients treated with ${ }^{125} \mathrm{I}$ BT combined with EBRT, when $\mathrm{RV}_{100}$ was $\leq 0.5 \mathrm{ml}$ [30]. Furthermore, reduction of rectal $\mathrm{D}_{1 \mathrm{cc}}$ and $\mathrm{D}_{2 \mathrm{cc}}$ (below ROC values) also demonstrated a significant reduction in acute and late GI toxicities. Adjusting for these confounding effects through multi-variable analysis maintained the reduced acute and late GI toxicity rates for patients with RS insertion, further supporting evidence from the current literature [4-6, 22, 27].

Therefore, the reported reduction in acute and late GI toxicities following RS insertion in this study reaffirms the results of current literature and strengthens understanding in the setting of ${ }^{125} \mathrm{I}$ LDR-BT monotherapy.

\section{Study strength and limitations}

The patients' demographics and underlying pathological features of diseases for the two investigated cohorts in this study were comparable in nature, except for cT stage, where a higher proportion of T2a patients were present in the non-RS cohort. However, there was no indication that $\mathrm{CT}$ stage was associated with a higher likelihood of GI toxicity, which minimized the probability of difference in cT stage as a strong confounding variable impacting on identified statistically significant difference in acute and late GI toxicities.

One significant advantage of this study was its' demonstration of association between reduced rectal dosimetry and acute and late GI toxicities. Furthermore, the statistical analyses allowed for an assessment of association between the effect of RS on GI toxicity outcomes, adjusting for rectal dosimetry values. This provided a new insight into the efficacy of RS in LDR-BT, which currently lacks sufficient evidence. Although, one limitation of this study was its' relatively small sample size, reducing the power of the study, and inability to detect statistically significant associations between RS and GI toxicities after adjusting for post-rectal $D_{1 c c}$ and $D_{2 c c}$ values, despite the positive effect of RS on reducing late GI toxicity, as shown by the magnitude of odds ratios from multi-variable analysis.

This multi-institutional approach used in this study, involving multiple community-based private radiation oncology centers, provided a larger sample size with a diverse, yet comparable patients' population, which allowed the data to be easily interpreted and applied by a wider variety of medical practitioners in their clinical practice. It also minimized issues of bias surrounding clinical practice, accessed barriers, and selection bias in single-institutional studies. Significantly, clinical practice, involving patient workup, imaging choice, and treatment modality, remained consistent across different campuses involved in this community-based study.

Despite efforts to minimize selection bias, the risk of bias still exists in this study due to the nature of its' design, which could be further minimized with a randomized clinical trial. The discretion of spacer implantation and choice of RS by the treating urologist was another major limitation of this study, which could be minimized with a blinded study with a 1:1 case-control randomization. 
The RS cohort had a shorter median follow-up duration when compared to the control cohort, which could contribute to the observed reduction in late GI toxicity outcomes. As such, a study with a longer duration of follow-up could further establish the effect of RS on late GI toxicity.

\section{Conclusions}

This Australian community-based retrospective study demonstrated that RS insertion with either HS or HA gel for treatment of low-risk and intermediate-risk PCa patients using ${ }^{125} \mathrm{I}$ LDR-BT was safe and effective in reducing rectal dosimetry as well as decreasing early GI toxicity. Our early data suggests that reduction in rectal dosimetry may translate to lower long-term GI toxicity. This approach also maintained comparable prostate dosimetry and similar rates of acute and late GU toxicities, when compared to patients not treated with a RS insertion. A randomized clinical trial could help further appreciate and quantify the risks and benefits of RS insertion in this clinical setting.

\section{Disclosure}

We declare one potential conflict of interest: the corresponding author Associate Professor Michael Chao is an advisory board member for Palette Life Sciences Pty Ltd.

The authors report no conflict of interest.

\section{References}

1. Bekelman JE, Rumble RB, Chen RC et al. Clinically localized prostate cancer: ASCO clinical practice guideline endorsement of an American Urological Association/American Society for Radiation Oncology/Society of Urologic Oncology guideline. J Clin Oncol 2018; 36: 3251-3258.

2. Pisansky TM, Thompson IM, Valicenti RK et al. Adjuvant and salvage radiation therapy after prostatectomy: ASTRO/ AUA guideline amendment, executive summary 2018. Pract Radiat Oncol 2019; 9: 208-213.

3. Price JG, Stone NN, Stock RG. Predictive factors and management of rectal bleeding side effects following prostate cancer brachytherapy. Int J Radiat Oncol Biol Phys 2013; 86: 842-847.

4. Van Gellekom MPR, Moerland MA, Van Vulpen M et al. Quality of life of patients after permanent prostate brachytherapy in relation to dosimetry. Int J Radiat Oncol Biol Phys 2005; 63: 772-780.

5. Snyder KM, Stock RG, Hong SM et al. Defining the risk of developing grade 2 proctitis following 125I prostate brachytherapy using a rectal dose-volume histogram analysis. Int J Radiat Oncol Biol Phys 2001; 50: 335-341.

6. Peters M, Hoekstra CJ, van der Voort van Zyp JRN et al. Rectal dose constraints for salvage iodine-125 prostate brachytherapy. Brachytherapy 2016; 15: 85-93.

7. Veccia A, Caffo O, Fellin G et al. Impact of post-implant dosimetric parameters on the quality of life of patients treated with low-dose rate brachytherapy for localised prostate cancer: results of a single-institution study. Radiat Oncol 2015; 10: 130.

8. Levy Y, Paz A, Yosef RB et al. Biodegradable inflatable balloon for reducing radiation adverse effects in prostate cancer. J Biomed Mater Res B Appl Biomater 2009; 91B: 855-867.

9. Noyes WR, Hosford CC, Schultz SE. Human collagen injections to reduce rectal dose during radiotherapy. Int J Radiat Oncol Biol Phys 2012; 82: 1918-1922.
10. Guimas V. Focal or whole-gland salvage prostate brachytherapy with iodine seeds with or without a rectal spacer for post-radiotherapy local failure: how best to spare the rectum? Brachytherapy 2016; 15: S199.

11. Melchert C, Gez E, Bohlen G et al. Interstitial biodegradable balloon for reduced rectal dose during prostate radiotherapy: Results of a virtual planning investigation based on the pre- and post-implant imaging data of an international multicenter study. Radiother Oncol 2013; 106: 210-214.

12. Schmid MP, Berger D, Heilmann $M$ et al. Inflatable multichannel rectal applicator for adaptive image-guided endoluminal high-dose-rate rectal brachytherapy: design, dosimetric characteristics, and first clinical experiences. J Contemp Brachytherapy 2017; 9: 359-363.

13. Taggar AS, Charas T, Weg E et al. Placement of an absorbable rectal hydrogel spacer in patients undergoing low-dose-rate brachytherapy with Pd-103 seeds. Brachytherapy 2017; 16: S64.

14. Prada PJ, Gonzalez H, Menéndez C et al. Transperineal injection of hyaluronic acid in the anterior perirectal fat to decrease rectal toxicity from radiation delivered with low-doserate brachytherapy for prostate cancer patients. Brachytherapy 2009; 8: 210-217.

15. Schörghofer A, Drerup M, Kunit T et al. Rectum-spacer related acute toxicity - endoscopy results of 403 prostate cancer patients after implantation of gel or balloon spacers. Radiat Oncol 2019; 14: 47.

16. Vanneste BGL, Pijls-Johannesma M, Van De Voorde L et al. Spacers in radiotherapy treatment of prostate cancer: Is reduction of toxicity cost-effective? Radiot Oncol 2015; 114: 276-281.

17. Heikkilä VP, Kärnä A, Vaarala MH. DuraSeal as a spacer to reduce rectal doses in low-dose rate brachytherapy for prostate cancer. Radiother Oncol 2014; 112: 233-236.

18. Pinkawa M, Escobar Corral N, Caffaro M et al. Application of a spacer gel to optimize three-dimensional conformal and intensity modulated radiotherapy for prostate cancer. Radiother Oncol 2011; 100: 436-441.

19. Pinkawa M, Piroth MD, Holy R et al. Spacer stability and prostate position variability during radiotherapy for prostate cancer applying a hydrogel to protect the rectal wall. Radiother Oncol 2013; 106: 220-224.

20. Susil RC, McNutt TR, DeWeese TL et al. Effects of prostate-rectum separation on rectal dose from external beam radiotherapy. Int J Radiat Oncol Biol Phys 2010; 76: 1251-1258.

21. Beydoun N, Bucci JA, Chin YS et al. First report of transperineal polyethylene glycol hydrogel spacer use to curtail rectal radiation dose after permanent iodine- 125 prostate brachytherapy. Brachytherapy 2013; 12: 368-374.

22. Mahal BA, Ziehr DR, Hyatt AS et al. Use of a rectal spacer with low-dose-rate brachytherapy for treatment of prostate cancer in previously irradiated patients: Initial experience and short-term results. Brachytherapy 2014; 13: 442-449.

23. Teh AYM, Ko HT, Barr G et al. Rectal ulcer associated with SpaceOAR hydrogel insertion during prostate brachytherapy. BMJ Case Reports 2014; 2014: bcr2014206931.

24. Uhl M, van Triest B, Eble MJ et al. Low rectal toxicity after dose escalated IMRT treatment of prostate cancer using an absorbable hydrogel for increasing and maintaining space between the rectum and prostate: Results of a multi-institutional phase II trial. Radiother Oncol 2013; 106: 215-219.

25. Ardekani MA, Ghaffari H. Optimization of prostate brachytherapy techniques with polyethylene glycol based hydrogel spacers: A systematic review. Brachytherapy 2020; 19: 13-23.

26. D'Amico AV, Whittington R, Malkowicz SB et al. Biochemical outcome after radical prostatectomy, external beam radiation therapy, or interstitial radiation therapy for clinically localized prostate cancer. JAMA 1998; 280: 969-974. 
27. Chao M, Spencer S, Guerrieri M et al. A single institution analysis of low-dose-rate brachytherapy: 5-year reported survival and late toxicity outcomes. J Contemp Brachytherapy 2018; 10: 155-161.

28. Thompson A, Keyes M, Pickles T et al. Evaluating the Phoenix definition of biochemical failure after 125I prostate brachytherapy: can PSA kinetics distinguish PSA failures from PSA bounces? Int J Radiat Oncol Biol Phys 2010; 78: 415-421.

29. Davis BJ, Horwitz EM, Lee WR et al. American Brachytherapy Society consensus guidelines for transrectal ultrasound-guided permanent prostate brachytherapy. Brachytherapy 2012; 11: 6-19.

30. Shiraishi Y, Yorozu A, Ohashi T et al. Dose constraint for minimizing grade 2 rectal bleeding following brachytherapy combined with external beam radiotherapy for localized prostate cancer: rectal dose-volume histogram analysis of 457 patients. Int J Radiat Oncol Biol Phys 2011; 81: e127-133.

31. Prada PJ, Fernández J, Martinez AA et al. Transperineal injection of hyaluronic acid in anterior perirectal fat to decrease rectal toxicity from radiation delivered with intensity modulated brachytherapy or EBRT for prostate cancer patients. Int J Radiat Oncol Biol Phys 2007; 69: 95-102.

32. Miller LE, Efstathiou JA, Bhattacharyya SK et al. Association of the placement of a perirectal hydrogel spacer with the clinical outcomes of men receiving radiotherapy for prostate cancer: a systematic review and meta-analysis. JAMA Netw Open 2020; 3: e208221-e.

33. Chao $\mathrm{M}, \mathrm{Ow} \mathrm{D}, \mathrm{Ho} \mathrm{H}$ et al. Improving rectal dosimetry for patients with intermediate and high-risk prostate cancer undergoing combined high-dose-rate brachytherapy and external beam radiotherapy with hydrogel space. J Contemp Brachytherapy 2019; 11: 8-13.

34. Rivard MJ, Coursey BM, DeWerd LA et al. Update of AAPM Task Group No. 43 Report: A revised AAPM protocol for brachytherapy dose calculations. Med Phys 2004; 31: 633-674.

35. Chao M, Bolton D, Lim Joon D et al. High dose rate brachytherapy boost for prostate cancer: Biochemical control and the impact of transurethral resection of the prostate and hydrogel spacer insertion on toxicity outcomes. J Med Imaging Radiat Oncol 2019; 63: 415-421.

36. Strom TJ, Wilder RB, Fernandez DC et al. A dosimetric study of polyethylene glycol hydrogel in 200 prostate cancer patients treated with high-dose rate brachytherapy \pm intensity modulated radiation therapy. Radiother Oncol 2014; 111: 126-131.

37. Trager M, Greenberger B, Harrison AS et al. SpaceOAR to improve dosimetric outcomes for monotherapy high-doserate prostate implantation in a patient with ulcerative colitis. J Contemp Brachytherapy 2018; 10: 577-582.

38. Kashihara T, Murakami N, Tselis N et al. Hyaluronate gel injection for rectum dose reduction in gynecologic high-doserate brachytherapy: initial Japanese experience. J Radiat Res 2019; 60: 501-508.

39. Hamstra DA, Mariados N, Sylvester J et al. Continued benefit to rectal separation for prostate radiation therapy: final results of phase III trial. Int J Radiat Oncol Biol Phys 2017; 97: 976-985.

40. Karsh LI, Gross ET, Pieczonka CM et al. Absorbable hydrogel spacer use in prostate radiotherapy: a comprehensive review of phase 3 clinical trial published data. Urology 2018; 115: 39-44.

41. Mariados N, Sylvester J, Shah D et al. Hydrogel spacer prospective multicenter randomized controlled pivotal trial: dosimetric and clinical effects of perirectal spacer application in men undergoing prostate image guided intensity modulated radiation therapy. Int J Radiat Oncol Biol Phys 2015; 92: 971-977.

42. van Gysen K, Kneebone A, Alfieri F et al. Feasibility of and rectal dosimetry improvement with the use of SpaceOAR ${ }^{\circledR}$ hydrogel for dose-escalated prostate cancer radiotherapy. J Med Imaging Radiat Oncol 2014; 58: 511-516.

43. Morita M, Fukagai T, Hirayama K et al. Placement of SpaceOAR hydrogel spacer for prostate cancer patients treated with iodine-125 low-dose-rate brachytherapy. Int J Urol 2020; 27: 60-66.

44. Liu H, Borden L, Wiant D et al. Proposed hydrogel-implant quality score and a matched-pair study for prostate radiation therapy. Pract Radiat Oncol 2020; 10: 202-208.

45. Patel A, Houser C, Benoit R et al. Patient-reported bowel quality of life with the combination of prostate external beam radiation, brachytherapy boost, and SpaceOAR. Brachytherapy 2019; 18: S74-S75.

46. Patel AK, Houser C, Benoit R et al. Acute patient-reported bowel quality of life and rectal bleeding with the combination of prostate external beam radiation, low-dose-rate brachytherapy boost, and SpaceOAR. Brachytherapy 2020; 19: 477-483.

47. Zhang H, Wang L, Riegel AC et al. Evaluation of rectal dose in prostate cancer patients having hydrogel spacer insertion in LDR brachytherapy and EBRT radiotherapy. Int J Radiat Oncol Biol Phys 2019; 105: E745.

48. Butler WM, Kurko BS, Scholl WJ et al. Effect of the timing of hydrogel spacer placement on prostate and rectal dosimetry of low-dose-rate brachytherapy implants. J Contemp Brachytherapy 2021; 13: 145-151.

49. Kahn J, Dahman B, McLaughlin C et al. Rectal spacing, prostate coverage, and periprocedural outcomes after hydrogel spacer injection during low-dose-rate brachytherapy implantation. Brachytherapy 2020; 19: 228-233.

50. Uhl M, Herfarth K, Eble MJ et al. Absorbable hydrogel spacer use in men undergoing prostate cancer radiotherapy: 12 month toxicity and proctoscopy results of a prospective multicenter phase II trial. Radiat Oncol 2014; 9: 96.

51. Wilder RB, Barme GA, Gilbert RF et al. Cross-linked hyaluronan gel reduces the acute rectal toxicity of radiotherapy for prostate cancer. Int J Radiat Oncol Biol Phys 2010; 77: 824-830.

52. Vaggers S, Rai BP, Chedgy ECP et al. Polyethylene glycol-based hydrogel rectal spacers for prostate brachytherapy: a systematic review with a focus on technique. World J Urol 2021; 39: 1769-1789.

53. Patel A, Houser C, Benoit R et al. Evaluation of dosimetric parameters and quality of life outcomes with use of SpaceOAR in patients undergoing prostate seed brachytherapy boost after external beam radiation. Brachytherapy 2018; 17: S80.

54. Chao M, Mario G, Chan Y et al. The use of HDR brachytherapy in prostate cancer: Can the urologist help in reducing GI and GU toxicity? Int J Urol 2019; 26: 184-185.

55. Wu SY, Boreta L, Wu A et al. Improved rectal dosimetry with the use of SpaceOAR during high dose rate brachytherapy. Brachytherapy 2017; 16: S109. 